\title{
RADIAL BASIS FUNCTION NETWORK ASSISTED WIDE-BAND BEAMFORMING
}

\author{
A. Wolfgang, S. Chen, L. Hanzo \\ School of ECS Univ. of Southampton, SO17 1BJ, UK. \\ Tel: +44-23-80-593 125, Fax: +44-23-80-594 508 \\ \{aw03r,sqc, 1h\}@ecs.soton.ac.uk \\ http: //www-mobile.ecs.soton.ac.uk
}

\begin{abstract}
In this paper we present a novel Radial Basis Function Network (RBFN) assisted wide-band beamformer which is based on the derivation of the Bayesian detector. The proposed receiver structure significantly outperforms conventional linear wide-band beamformers in terms of the achievable angular resolution and thus the receiver is capable of supporting a higher number of users. For the further enhancement of the BER performance and for the sake of complexity reduction a decision feedback aided RBF scheme is proposed.
\end{abstract}

\section{INTRODUCTION}

During the last decade multiple antenna based beamformers [1] have been the subject of intense research, since they are capable of providing a substantial increase of the achievable system capacity. Beamforming receivers operating in a wide-band scenario, where the different users' signals received by the base station are subject to dispersion-induced Inter Symbol Interference (ISI) also have to perform time domain equalisation in addition to angular-domain beamforming. Such receivers are often referred to as space-time equalisers or wide-band beamformers.

A classic assumption in the field of multiple antenna based beamformers is that the number of receiver antennas is at least as high as the number of users supported, because the performance of 'over-loaded' beamformers tends to drop dramatically. This is due to the fact that as the number of users increases, the detection problem which has to be solved becomes linearly non-separable. Hence the family of receivers based on linear combiners are subject to a severe performance degradation. As a consequence, numerous non-linear beamforming schemes [2-4] mostly based on Radial Basis Function Networks (RBFN) [5] have been proposed in the literature.

Similar developments were observed also in the field of channel equalisation. The lack of linear separability at the channel's output induced by the ISI has led to the intensive investigation of RBFN assisted equalisers. In [6] it has been shown that RBFN equalisers are capable of combating both the ISI and the co-channel interference in single-antenna systems. Furthermore, RBF-aided receivers may also be used for separating multiple users based on their unique, user specific Channel Impulse Response (CIR).

The substantial performance advantage of non-linear beamformers designed for ISI-free narrow-band channels as well as the success of non-linear channel equalisers designed for single-user systems has motivated our research to introduce a combined nonlinear space-time equaliser. It is important to underline here that the multiple antennas are not used for increasing the throughput of

The financial support of the EU under the auspices of the Phoenix project is gratefully acknowledged. The authors are also grateful to their colleagues for the enlightenment gained within the Phoenix consortium. a single user but rather for separating multiple users. Thus each of the users communicates over a single-input multiple-output (SIMO) channel. This will become clear from the system model presented in Section 2. The remainder of the paper is organised as follows. In Section 3 we derive the Bayesian beamforming solution, which can be implemented using a RBFN and we will present a linear Minimum Mean Squared Error (MMSE) receiver, which serves as our bench-marker. The performance of the RBFN assisted wide-band beamformer is investigated in terms of its Bit Error Ratio (BER) performance over a static multi-path channel in comparison to the linear MMSE bench marker. In Section 4 the proposed scheme is enhanced by introducing a decision feedback structure for the sake of further performance improvements and complexity reduction. Our conclusions are offered in Section 5.

\section{SYSTEM MODEL}

The system ${ }^{1}$ considered consists of $Q$ users modelled as Binary Phase Shift Keying (BPSK) modulated point-sources in the far field of the receiver associated with a different angle of incidence $\theta_{q}$, as seen in Figure 1. The channel experienced by each user's signal is modelled as a Finite Impulse Response (FIR) filter described as

$$
h_{q}(z)=\sum_{k=0}^{K_{q}} h_{q, k} z^{-k},
$$

where $K_{q}+1$ and $h_{q, k}$ are the filter length and the tap weights of the $q^{t h}$ source' channel. The CIR of all users is normalised to unit energy. Additionally, we consider a one-dimensional $L$-element linear array of omni-directional antennas having an inter-element spacing of $d=\frac{\lambda}{2}$, where $\lambda$ is the wave-length of the sources. Each of the antenna elements is followed by a tapped delay line of length $m$, which is also referred to as the feed-forward section of the receiver. The channel output of all array elements is expressed by the super-vector $\mathbf{v}_{k}=\left[\boldsymbol{v}_{k}^{T}, \ldots, \boldsymbol{v}_{k-m+1}^{T}\right]^{T}$, where $\boldsymbol{v}_{k}$ is a column vector having $L$ elements, with $v_{l, k}$ representing the output signal of array element $l$ at time instant $k$.

In order to arrive at an expression for the channel output $\mathbf{v}_{k}$ we first introduce the coefficient $f_{l q, k}$, which is given as

$$
f_{l q, k}=A_{q} e^{j \omega t_{l}\left(\theta_{q}\right)} h_{q, k},
$$

where $A_{q}$ is the amplitude of the signal received from the $q^{t h}$ source, $h_{q, k}$ is the channel coefficient of user $q$ at time instant $k$ and $t_{l}\left(\theta_{q}\right)$ is the relative time delay of the signal transmitted by the $q^{t h}$ source at the $l^{t h}$ array element. We denote furthermore the

\footnotetext{
${ }^{1}$ Throughout this work the variables which are used for representing vectors and matrices are printed in italic and bold. Super-matrices and super-vectors are represented by bold letters.
} 


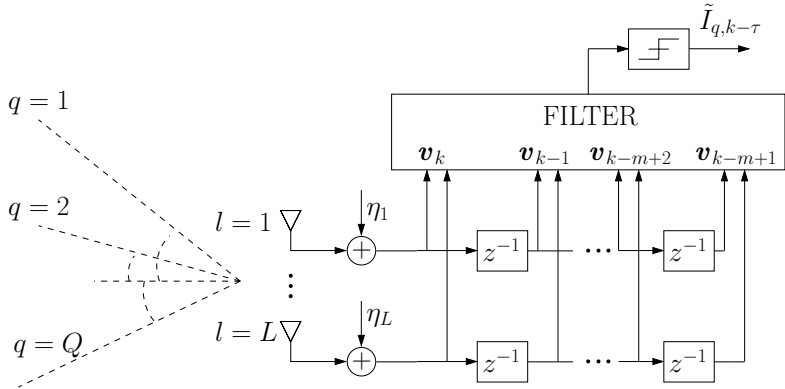

Figure 1: Space-time receiver for $Q$ users with angle of incidence $\theta_{q}$ employing a $L$-element linear antenna array. Each of the elements is followed by a delay line consisting of $m$ taps.

$(L \times Q)$-dimensional matrix $\boldsymbol{F}_{k}$ as

$$
\boldsymbol{F}_{k}=\left(\begin{array}{ccc}
f_{11, k} & \cdots & f_{1 Q, k} \\
\vdots & & \vdots \\
f_{L 1, k} & \cdots & f_{L Q, k}
\end{array}\right)
$$

The super-matrix $\mathbf{F}$, which represents the complete system is obtained by concatenating the $(L \times Q)$-dimensional matrices $\boldsymbol{F}_{k}$, yielding:

$$
\mathbf{F}=\left(\begin{array}{cccccc}
\boldsymbol{F}_{k} & \cdots & \boldsymbol{F}_{k-m+1} & 0 & \cdots & 0 \\
0 & \boldsymbol{F}_{k} & \cdots & \boldsymbol{F}_{k-m+1} & \ddots & \vdots \\
\vdots & \ddots & \ddots & \ddots & \ddots & 0 \\
0 & \cdots & 0 & \boldsymbol{F}_{k} & \cdots & \boldsymbol{F}_{k-m+1}
\end{array}\right)
$$

The channel output vector $\mathbf{v}_{k}$ can now be expressed as

$$
\begin{aligned}
\mathbf{v}_{k} & =\mathbf{F}\left[\boldsymbol{I}_{k}^{T}, \ldots, \boldsymbol{I}_{k-m+1}^{T}\right]^{T}+\left[\boldsymbol{\eta}_{1, k}^{T}, \ldots, \boldsymbol{\eta}_{L, k}^{T}\right]^{T} \\
& =\mathbf{F I}_{k}+\boldsymbol{\eta}_{k} \\
& =\overline{\mathbf{v}}_{k}+\boldsymbol{\eta}_{k},
\end{aligned}
$$

where $\boldsymbol{I}_{k}=\left[I_{1, k}, \ldots, I_{Q, k}\right]^{T}$ is a column vector containing the symbols transmitted by the $Q$ sources and $\boldsymbol{\eta}_{l, k}=$ $\left[\eta_{l, k}, \ldots \eta_{l, k-m+1}\right]^{T}$ is the complex valued Additive White Gaussian Noise (AWGN) vector having a variance of $E\left[\left|\eta_{l, k}\right|^{2}\right]=$ $2 \sigma^{2}$. The Signal to Noise Ratio (SNR) of the $q^{\text {th }}$ user's signal at the antenna array's output can be written as

$$
\mathrm{SNR}_{q}=\frac{A_{q}^{2}}{2 \sigma^{2}},
$$

and the Signal to Interference Ratio $\left(\mathrm{SIR}_{q}\right)$ of the desired user $q=$ 1 with respect to the interfering source $q$ is given as

$$
\operatorname{SIR}_{q}=\frac{A_{1}^{2}}{A_{q}^{2}}
$$

\section{RECEIVER STRUCTURES}

In this section different implementations of the filter employed by the receiver depicted in Figure 1 are introduced. For notational convenience we assume that the CIRs of all users have an equal length $\left(K_{q}=K\right)$. First we will provide a closed-form expression for the weights of a linear receiver, which minimises the MSE at the output of the detector. This MMSE receiver will serve as a bench-marker for the optimal Bayesian wide-band beamformer, which can be implemented using a RBFN.

\subsection{Linear Receiver}

Under the assumption that user $q$ is the desired user, the weight vector $\mathbf{w}_{q}$ of a linear filter which combines the signals of the $L$ array elements can be readily written as [7]:

$$
\mathbf{w}_{q}=\left(\mathbf{F F}^{H}+2 \sigma^{2} \mathcal{I}_{L m}\right)^{-1} \mathbf{F}(:, Q \tau+q),
$$

where $\mathcal{I}_{L m}$ is the $(L m \times L m)$ identity matrix, $\mathbf{F}(:, Q \tau+q)$ is the $(Q \tau+q)^{t h}$ column of the matrix $\mathbf{F}$ and $\tau$ is the decision delay used for the detection of the $q^{t h}$ user's transmitted symbol with $0 \leq \tau \leq m+K-1$. The $q^{t h}$ user's detected symbol can now be written as

$$
\tilde{I}_{q, k-\tau}=\mathbf{w}_{q}^{H} \mathbf{v}_{\mathbf{k}}
$$

where ${ }^{H}$ denotes the Hermitian (conjugate transpose) of a vector.

\subsection{Bayesian Receiver}

The derivation of the Bayesian receiver is based on the different possible noiseless channel output vectors $\overline{\mathbf{v}}_{k}$. For BPSK modulated sources there exist

$$
n_{s}=2^{Q m+\sum_{q} K_{q}}=2^{Q(m+K)}
$$

number of possible noiseless output phasors $\overline{\mathbf{v}}_{k}$. Denote each of the $n_{s}$ possible combinations of the input sequence $\mathbf{I}_{k}$ of length $Q(m+K)$ as $\mathbf{s}_{i}, 1 \leq i \leq n_{s}$, where the channel input state $\mathbf{s}_{i}$ determines the desired channel output state $\mathbf{r}_{i}, 1 \leq i \leq n_{s}$. In mathematical terms this can be written as

$$
\overline{\mathbf{v}}_{k}=\mathbf{r}_{i} \quad \text { if } \quad \mathbf{I}_{k}=\mathbf{s}_{i},
$$

with $\mathbf{r}_{i}=\mathbf{F} \mathbf{s}_{i}$. The set of all possible desired output states can be partitioned into two subsets depending on the binary value of the transmitted symbol $I_{q, k-\tau}$ of the desired user $q$ as

$$
\begin{aligned}
& \mathcal{V}^{q,+}=\left\{\overline{\mathbf{v}}_{k} \mid I_{q, k-\tau}=+1\right\} \\
& \mathcal{V}^{q,-}=\left\{\overline{\mathbf{v}}_{k} \mid I_{q, k-\tau}=-1\right\} .
\end{aligned}
$$

The number of vectors $\overline{\mathbf{v}}_{k}$ in $\mathcal{V}^{q,+}$ and $\mathcal{V}^{q,-}$ are $n_{s}^{q,+}$ and $n_{s}^{q,-}$, respectively. Note that $n_{s}^{q,+}=n_{s}^{q,-}=n_{s} / 2$ for all $q$. The optimal Bayesian solution for the receiver structure shown in Figure 1 can now be written as

$$
\tilde{I}_{q, k-\tau}=\operatorname{sgn}\left(f_{B, q}\left(\mathbf{v}_{k}\right)\right)=\left\{\begin{array}{ll}
+1 & \text { if } f_{B, q}\left(\mathbf{v}_{k}\right) \geq 0 \\
-1 & \text { if } f_{B, q}\left(\mathbf{v}_{k}\right)<0
\end{array},\right.
$$

where the optimal Bayesian decision function $f_{B, q}(\cdot)$ based on the difference of the associated conditional density functions is given as

$$
\begin{aligned}
f_{B, q}\left(\mathbf{v}_{k}\right)= & P\left(\mathbf{v}_{k} \mid I_{q, k-\tau}=+1\right)-P\left(\mathbf{v}_{k} \mid I_{q, k-\tau}=-1\right) \\
= & \sum_{i=1}^{n_{s}^{q,+}} p_{i}^{+} p\left(\mathbf{v}_{k}-\mathbf{r}_{i}^{q,+}\right)-\sum_{i=1}^{n_{s}^{q,-}} p_{i}^{-} p\left(\mathbf{v}_{k}-\mathbf{r}_{i}^{q,-}\right) \\
= & \sum_{i=1}^{n_{s}^{q,+}} p_{i}^{+} \frac{1}{\left(2 \pi \sigma^{2}\right)^{-L m}} \exp \left(-\frac{\left\|\mathbf{v}_{k}-\mathbf{r}_{i}^{q,+}\right\|^{2}}{2 \pi \sigma^{2}}\right) \\
& -\sum_{i=1}^{n_{s}^{q,-}} p_{i}^{-} \frac{1}{\left(2 \pi \sigma^{2}\right)^{-L m}} \exp \left(-\frac{\|\left(\mathbf{v}_{k}-\mathbf{r}_{i}^{q,-} \|^{2}\right.}{2 \sigma^{2}}\right),
\end{aligned}
$$




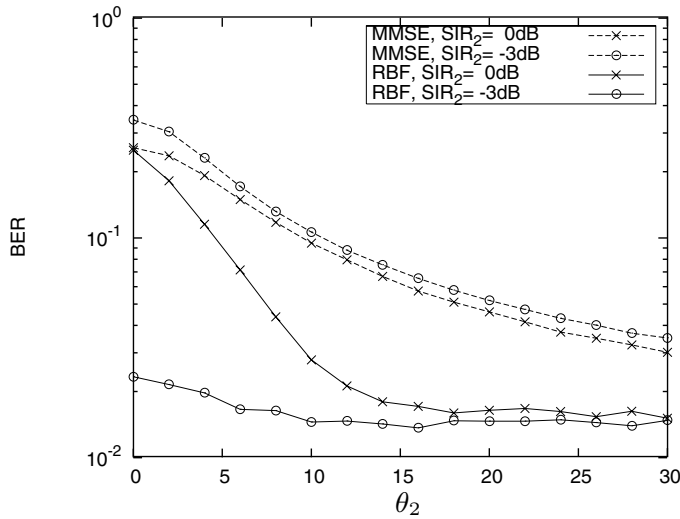

Figure 2: BER of user $q=1$ versus the angle of incidence $\theta_{2}$ of user two for different SIRs. The desired source was fixed to $\theta_{1}=0^{\circ}$ and $\mathrm{SNR}=10 \mathrm{~dB}$, the receiver employed two antenna elements and the feed-forward order was chosen to be $m=5$. The decision delay was $\tau=1$.

where $\mathbf{r}_{i}^{q,+} \in \mathcal{V}^{q,+}, \mathbf{r}_{i}^{q,-} \in \mathcal{V}^{q,-}$ and $p_{i}^{+}$and $p_{i}^{-}$are the a-priori probabilities of $\mathbf{r}_{i}^{q,+}$ and $\mathbf{r}_{i}^{q,-}$, respectively.

\subsection{RBF Receiver}

The overall response of a RBFN can be formulated as [5]

$$
\begin{array}{r}
f_{R B F}\left(\mathbf{v}_{k}\right)=\sum_{i=1}^{N_{c}} w_{i} \phi\left(\mathbf{v}_{k}, \mathbf{c}_{i}\right), \\
\phi\left(\mathbf{v}_{k}, \mathbf{c}_{i}\right)=\exp \left(-\frac{\left\|\mathbf{v}_{k}-\mathbf{c}_{i}\right\|^{2}}{\rho}\right),
\end{array}
$$

where $\mathbf{c}_{i}$ represents the RBF centres, which have the same dimensionality as the input vector $\mathbf{v}_{k}, \phi(\cdot)$ is the radial basis function chosen to be the Gaussian function, $N_{c}$ is the number of centres, $\rho$ is the radius or spread of the RBF and $w_{i}$ are the RBF weights. The detected symbol of user $q$ after the slicer can be expressed as

$$
\tilde{I}_{q, k-\tau}=\operatorname{sgn}\left(f_{R B F}\left(\mathbf{v}_{k}\right)\right) .
$$

The relationship between the RBF network and the Bayesian solution can be established by setting the RBF centres $\mathbf{c}_{i}, i=$ $1, \ldots, N_{c}=n_{s}$ to the possible noise free channel output vectors $\overline{\mathbf{v}}_{k}$ and setting the weight $w_{i}$ to +1 if $\mathbf{c}_{i} \in \mathcal{V}^{q,+}$ and to -1 if $\mathbf{c}_{i} \in \mathcal{V}^{q,-}$. The RBF spread $\rho$ is set to the noise variance $2 \sigma^{2}$. Given this configuration, the RBF assisted receiver realises the Bayesian receiver.

\subsection{Simulations}

In this subsection we compare the performance of the linear MMSE beamformer and the RBF assisted beamformer. For both systems we assume that the channel of each user is known, therefore the MMSE weights can be calculated using the closed form expression of Equation (7). In our simulations we used a twoelement linear array supporting two users communicating over the identical two-tap channels $h_{1}(z)=h_{2}(z)=\sqrt{0.5}+\sqrt{0.5} z^{-1}$ having equal-power taps. The SNR of the desired user was fixed to $10 \mathrm{~dB}$. The feed-forward order was chosen to be $m=5$ and the decision delay was set to $\tau=1$. In Figure 2 the BER of user $q=1$ versus the angle of incidence $\theta_{2}$ of user two is shown for different SIRs. The desired user's angle of incidence was $\theta_{1}=0^{\circ}$.

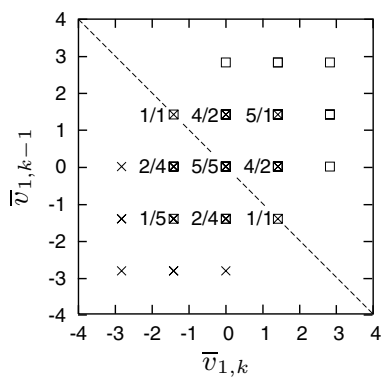

(a) $\mathrm{SIR}_{2}=0 \mathrm{~dB}$

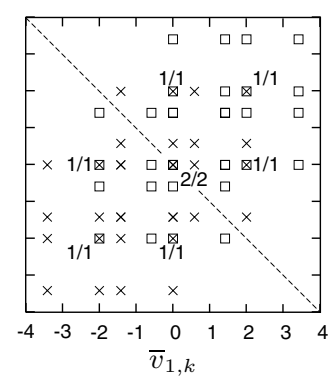

(b) $\mathrm{SIR}_{2}=-3 \mathrm{~dB}$
Figure 3: Noiseless channel output constellation at the input of the receiver without noise for $Q=2, \theta_{1}=\theta_{2}=0^{\circ}$, $m=2$ and $\tau=1$ at array element $l=1$. The legend $\square$ marks $I_{1, k-\tau}=+1$ and the $\times$ indicates $I_{1, k-\tau}=$ -1 . The numbers next to the states, which host more than one phasor indicate the ratio $n_{i}^{+} / n_{i}^{-}$as defined in Equation (15) and the dashed line represents the linear decision boundary.

It can be seen that the MMSE receiver converges only for large angular separations between the two users and has a residual BER of $P_{e, r e s}=\frac{1}{2^{(K+m)}}$ [5]. As the power of the interferer increases, the performance of the linear wide-band beamformer degrades. By contrast, the non-linear RBF receiver is capable of converging towards the above-mentioned residual BER at a significantly smaller angular separation between the two sources. It can also be observed in Figure 2 that for $\mathrm{SIR}_{2}=-3 \mathrm{~dB}$ the receiver is capable of differentiating between the two sources for a very small $\theta_{2}$.

The unconventional behaviour of having a better BER performance at the lower SIR of $-3 \mathrm{~dB}$ can be explained by considering Figure 3, which shows the noiseless beamformer input phasors $\left[\bar{v}_{1, k-1} \bar{v}_{1, k-1}\right]^{T}$ for $\theta_{1}=\theta_{2}=0^{\circ}, m=2$ and $\tau=1$. The states were calculated using Equation (4) for all $n_{s}$ possible different transmitted symbol sequences $\mathbf{s}_{i}$. In Figure 3(a) it can be seen that for $\mathrm{SIR}_{2}=0 \mathrm{~dB}$ many of the $n_{s}=2^{Q(m+K)}=64$ phasors are merged together in the observation space, thus resulting in only 19 different states. The bit error probability contribution of each state can be expressed for $S N R \rightarrow \infty$ as

$$
P_{e, i}^{\mathrm{RBFN}}=\frac{n^{+}}{n_{s}}\left(1-\frac{n^{+}}{n^{-}+n^{+}}\right),
$$

where $n_{i}^{+}$and $n_{i}^{-}$are the number of phasors corresponding to a transmitted +1 and -1 , respectively, which result in the same channel output state $i$. In our example this would yield $P_{e}^{\mathrm{RBFN}}=$ $\sum_{i=1}^{19} P_{e, i}^{\mathrm{RBFN}}=\frac{8.5}{32}=0.27$. The BER of the MMSE beamformer can be calculated by summing up the a-priori probabilities of all states, which are wrongly classified by the linear decision boundary, yielding $P_{e}^{\mathrm{MMSE}}=\frac{5+0.5 * 7}{32}=0.27$.

However, when the power of the interferer is increased, yielding $\mathrm{SIR}_{2}=-3 \mathrm{~dB}$, more constellation points become separable in Figure $3(\mathrm{~b})^{2}$ resulting in only 7 states, which host phasors corresponding to both a transmitted +1 and to -1 . In fact, these 7 states are causing the residual BER, as it can be seen from $P_{e}^{\mathrm{RBFN}}=\sum_{i=1}^{49} P_{e, i}^{\mathrm{RBFN}}=\frac{4}{32}=P_{e, \text { res }}=0.13$. A MMSE beamformer results in a BER of $P_{e}^{\mathrm{MMSE}}=\frac{9+0.5 * 2}{32}=0.31$.

Interpreting the results of Figure 2 further, one may argue that the MMSE receiver treats the co-channel interference as additional noise, whereas the RBF assisted detector attempts to detect the

\footnotetext{
${ }^{2}$ States that host multiple phasors corresponding to the same transmitted symbol $I_{1, k-\tau}$ are not indicated with $n_{i}^{+} / n_{i}^{-}$owing to lack of space. The states can however be identified by comparison with Figure 3(a).
} 


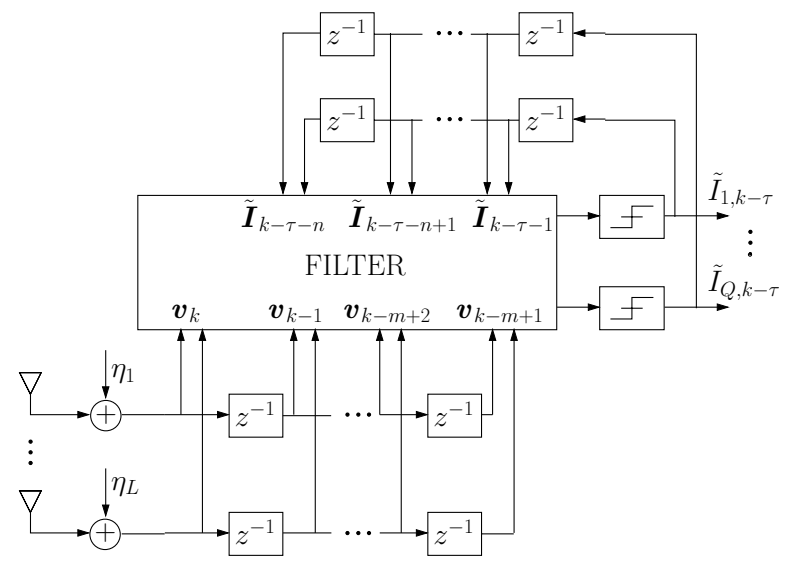

Figure 4: General decision feedback space-time receiver with a $L$-element linear antenna array. Each of the elements is followed by a delay line consisting of $m$ taps. $n$ previously detected symbols of each user are used as feedback for the receiver.

received phasor constellation more intelligently. The associated performance improvement however is achieved at a significantly increased computational cost, which will be reduced in the next section.

\section{DECISION FEEDBACK AIDED BEAMFORMER}

The performance of both linear and non-linear equalisers can be enhanced by incorporating a decision feedback structure [5] in the receiver, as shown in Figure 4. In addition to the feed-forward order $m$ and the decision delay parameter $\tau$ we introduce the decision feedback order $n$. Note, that the oldest symbol vector, which still influences the detected symbol $\tilde{I}_{q, k-\tau}$ is $\boldsymbol{I}_{k-m+1-K}$. Furthermore, the oldest feedback symbol vector is $\boldsymbol{I}_{k-\tau-n}$. Without loss of generality we therefore chose $n=m+K-1-\tau$ for the derivation of the proposed decision feedback aided RBF beamformers. In order to describe the feedback structure, we first divide the system matrix $\mathbf{F}$ into two sub-matrices $[5]^{3}$

$$
\mathbf{F}=\left[\begin{array}{ll}
\mathbf{F}_{1} & \mathbf{F}_{2}
\end{array}\right],
$$

where $\mathbf{F}_{1}$ hosts the first $Q(\tau+1)$ columns of $\mathbf{F}$ and $\mathbf{F}_{2}$ represents the last $Q n$ columns in $\mathbf{F}$. The array output can then be written as

$$
\begin{aligned}
\mathbf{v}_{k} & =\mathbf{v}_{k}^{f f}+\mathbf{v}_{k}^{f b} \\
& =\overline{\mathbf{v}}_{k}^{f f}+\overline{\mathbf{v}}_{k}^{f b}+\boldsymbol{\eta}_{k} \\
& =\mathbf{F}_{1} \mathbf{I}_{k}^{f f}+\mathbf{F}_{2} \mathbf{I}_{k}^{f b}+\boldsymbol{\eta}_{k},
\end{aligned}
$$

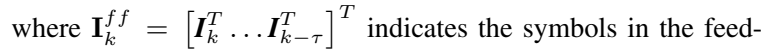
forward shift register and $\mathbf{I}_{k}^{f b}=\left[\boldsymbol{I}_{k-\tau-1}^{T} \ldots \boldsymbol{I}_{k-\tau-n}^{T}\right]^{T}$ denotes the symbols in the feedback register. The complexity reduction achieved by the decision feedback is due to the fact that the previously received symbols of all users have already been decided upon and hence their ambiguity imposed on the constellation may be eliminated.

${ }^{3}$ If $n$ is chosen differently, the system matrix has to be divided into three sub-matrices in order to derive the proposed algorithms [5].

\subsection{Linear Decision Feedback Receiver}

Following the approach presented in [8], the feed-forward weights $\mathbf{w}_{q}^{f f}$ and the feedback weights $\mathbf{w}_{q}^{f b}$ of the $q^{\text {th }}$ user can be written as [8]

$$
\begin{aligned}
& \mathbf{w}_{q}^{f f}=\left(\mathbf{F}_{1} \mathbf{F}_{1}^{H}+2 \sigma^{2} \mathcal{I}_{L m}\right)^{-1} \mathbf{F}(:, Q \tau+q) \\
& \mathbf{w}_{q}^{f b}=\mathbf{F}_{2}^{H} \mathbf{w}_{q}^{f f},
\end{aligned}
$$

where $\mathbf{F}(:, Q \tau+q)$ is the $(Q \tau+q)^{t h}$ column of $\mathbf{F}$. The detected symbol $\tilde{I}_{q, k-\tau}$ can then be written as

$$
\begin{aligned}
\tilde{I}_{q, k-\tau} & =\left(\mathbf{w}_{q}^{f f}\right)^{H} \mathbf{v}_{k}^{f b}-\left(\mathbf{w}_{q}^{f b}\right)^{H}\left[\tilde{\mathbf{I}}_{k-\tau-1}^{T} \ldots \tilde{\boldsymbol{I}}_{k-\tau-n}^{T}\right]^{T} \\
& =\left(\mathbf{w}_{q}^{f f}\right)^{H} \mathbf{v}_{k}^{f f}-\left(\mathbf{w}_{q}^{f b}\right)^{H} \tilde{\mathbf{I}}_{k}^{f b}
\end{aligned}
$$

where $\tilde{\boldsymbol{I}}_{k}$ was defined in Equation (4). Note, that in order to be able to perform decision feedback all users' signal has to be detected.

\subsection{RBF Decision Feedback Receiver}

In this subsection two different implementations of the Bayesian decision feedback aided wide-band beamformer are presented, which are again based on the noise-free channel output states. Both the proposed sub-centre selection [5] and the spacetranslation [5] approach realize the same beamformer.

Sub-centre Selection [5](Figure 8.20): The binary subsets $\mathcal{V}^{q,+}$ and $\mathcal{V}^{q,-}$ given in Equation (11) can be further partitioned into $n_{f b}$ subsets $\mathcal{V}_{j}^{q,+}$ and $\mathcal{V}_{j}^{q,+}$ according to the $n_{f b}$ possible feedback states $\mathbf{s}_{j}^{f b}, 1 \leq j \leq n_{f b}$ of the feedback shift register, where

$$
n_{f b}=2^{Q n} \text {. }
$$

The union of all the $n_{f b}$ number of feedback states associated with the two legitimate binary symbols transmitted by user $q$ can then be written as

$$
\mathcal{V}^{q, \pm}=\bigcup_{1 \leq j \leq n_{f b}} \mathcal{V}_{j}^{q, \pm}
$$

where $\mathcal{V}_{j}^{q, \pm}$ is the set of possible values of the noise-free channel output vectors $\overline{\mathbf{v}}_{k}$ associated with the delayed transmitted symbol of the desired user $I_{q, k-\tau}$ and the feedback symbol sequence $\tilde{\mathbf{I}}_{k}^{f b}$. This yields

$$
\mathcal{V}_{j}^{q, \pm}=\left\{\overline{\mathbf{v}}_{k} \mid I_{q, k-\tau}= \pm 1 \cap \tilde{\mathbf{I}}_{k}^{f b}=\mathbf{s}_{j}^{f b}\right\} .
$$

The role of the feedback structure in the receiver is now to select a reduced-size subset of particular RBF centres for a given feedback vector $\tilde{\mathbf{I}}_{k}^{f b}$ on the basis of the already decided symbols, since this allows us to reduce the detector's complexity. Explicitly, if the feedback vector is $\tilde{\mathbf{I}}_{k}^{f b}=\mathbf{s}_{j}^{f b}$, then the noiseless channel output vectors $\mathbf{r}_{j, i}^{ \pm} \in \mathcal{V}_{j}^{ \pm}$are selected as centres of the RBFN. The weights $w_{i}$ of the network are set to +1 if we have $\mathbf{r}_{j, i} \in \mathcal{V}_{j}^{+}$and to -1 if $\mathbf{r}_{j, i} \in \mathcal{V}_{j}^{-}$. With the advent of the decision feedback the number of $\mathrm{RBF}$ centres is reduced to

$$
N_{c}^{f b}=2^{Q(m+K-n)}=2^{Q(\tau+1)} .
$$

Space Translation [5](Figure 8.27): The implementation of the sub-centre selection based feedback structure is not particularly attractive, since $n_{f b}$ different sets of RBF centres have to be stored. Therefore the approach often used for RBF assisted DFEs [5] is adopted, which interprets the decision feedback as a 'space translation' of the input space vector $\mathbf{v}_{k}$ to $\mathbf{v}_{k}^{\prime}$. Under the assumption that the feedback vector is correct, Equation (17) can be re-written as

$$
\mathbf{v}_{k}^{\prime}=\mathbf{v}_{k}-\mathbf{F}_{2} \tilde{\mathbf{I}}_{k}^{f b}=\mathbf{F}_{1} \tilde{\mathbf{I}}_{k}^{f f},
$$




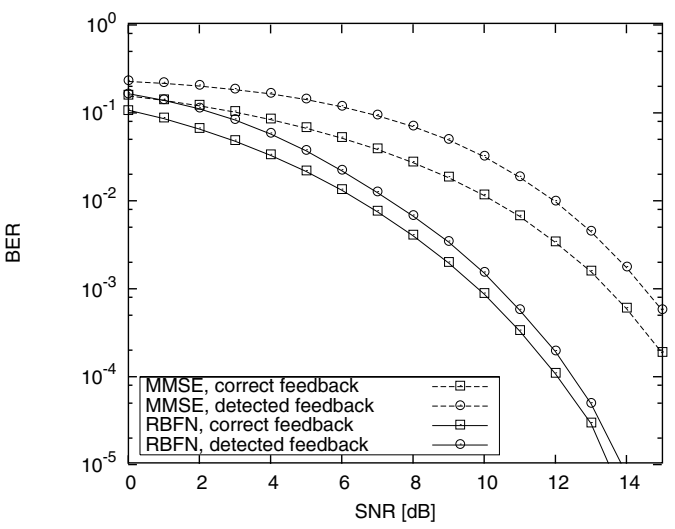

Figure 5: BER of user $q=1$ versus SNR for a scenario employing 2 users $\left(\theta_{1}=0^{\circ}, \theta_{2}=15^{\circ}\right)$ received with equal power. The simulation were performed for correct fed back symbols and detected fed back symbols. The receiver employed two receive antennas and used $m=2$, $\tau=1$ and $n=1$.

where $\mathbf{v}_{k}^{\prime}$ is the observation space owing to the decision feedback. The number of possible states in this new observation space is $n_{f b, s}=2^{Q(\tau+1)}=N_{c}^{f b}$. The configuration of the RBF network is obtained following the approach used for the derivation of the conventional RBF space-time equaliser of Sections 3.2 and 3.3 for the new reduced-size observation space $\mathbf{v}_{k}^{\prime}$. This structure of the RBF receiver has the advantage that only the beamformer's input vector $\mathbf{v}_{k}$ has to be transformed into $\mathbf{v}_{k}^{\prime}$ according to Equation (25) and the RBF centres remain unchanged. For the detection of $\tilde{I}_{1, k-\tau}$ the received signal vector $\mathbf{v}_{k}$ is transformed into the translated space by subtracting the product of the feedback sequence $\tilde{\mathbf{I}}_{k}^{f b}$ and $\mathbf{F}_{2}$, as described by Equation (25). The centres of the decision feedback aided RBF network using 'space translation' are determined by $\mathbf{F}_{1} \mathbf{s}_{i}, 1 \leq i \leq n_{f b, s}$, where $\mathbf{s}_{i}$ represents the possible transmitted symbol sequences of length $Q(\tau+1)$.

\subsection{Simulations and Results}

In order to investigate the effect of error propagation caused by the decision feedback, we investigated the performance of a beamformer employing two antennas for a constellation associated with two users $\left(\theta_{1}=0^{\circ}, \theta_{2}=15^{\circ}\right)$, whose signals were received at an equal power. The two-tap channels of the users were again chosen to be $h_{1}(z)=h_{2}(z)=\sqrt{0.5}+\sqrt{0.5} z^{-1}$ and the beamformer parameters were set to $m=2, n=1$ and $\tau=1$. The graphs in Figure 5 show that the RBF assisted beamformer outperforms the linear MMSE beamformer, illustrating also that the error propagation induced by the feedback structure has a less detrimental effect on the $\mathrm{RBF}$ receiver.

The increased angular separability of the RBF receiver compared to that of the MMSE receiver, which was shown in Figure 2 in the absence of decision feedback is also valid for the structure employing a feedback. An increased angular resolution should thus lead to an increased number of users that may be supported by the system at a given BER. Figure 6 shows the BER of the desired user $q=1$ for a two-element beamformer supporting three users received at equal power over the channel characterised by $h_{1}(z)=h_{2}(z)=h_{3}(z)=\sqrt{0.5}+\sqrt{0.5} z^{-1}$. The angles of incidence were chosen to be $\theta_{1}=0^{\circ}, \theta_{2}=15^{\circ}$ and $\theta_{3}=-30^{\circ}$. It is clearly illustrated that the RBF receiver is capable of detecting the desired user $q=1$ even in this 'over-loaded' scenario. If the feed-forward order $m$ and the decision delay $\tau$ are chosen to be sufficiently high, the RBF receiver is capable of approaching

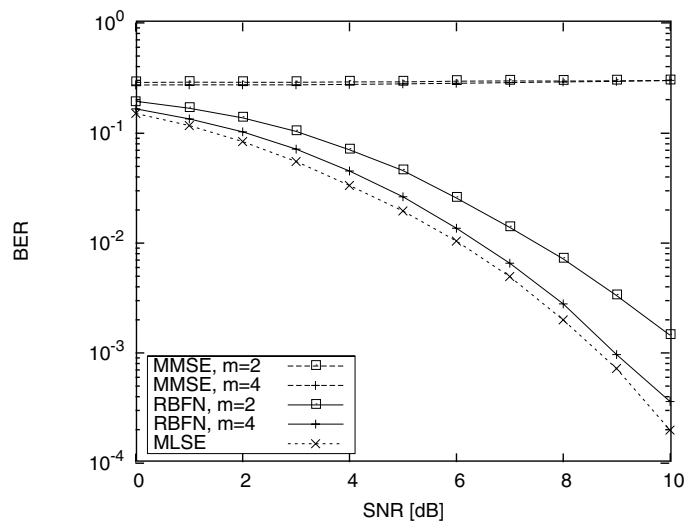

Figure 6: BER of user $q=1$ versus SNR for a scenario supporting 3 users received at equal power. The receiver parameters were: $L=2, \tau=m-1, n=m+K-1-\tau$ and $m$ is given in the legend.

the performance of the Maximum Likelihood Sequence Estimator (MLSE), which was implemented using the classic Viterbi algorithm. By contrast, the MMSE beamformer is incapable of adequate operation in an over-loaded scenario.

\section{CONCLUSIONS AND CURRENT RESEARCH}

A novel Bayesian wide-band beamformer has been investigated both with and without decision feedback, which has been implemented using a RBFN. The beamformer's performance was compared to both existing linear combiners and to the optimum MLSE beamformer. It has been shown that the proposed non-linear structure is capable of outperforming linear receivers, especially in overloaded systems. Our future work will focus on further complexity reduction of the RBFN assisted wide-band beamformer and on its adaptive implementations designed for time-variant channels.

\section{REFERENCES}

[1] J.S. Blogh, L. Hanzo: Third-Generation Systems and Intelligent Wireless Networking - Smart Antennas and Adaptive Modulation, John Wiley, 2002.

[2] A.H. El Zooghby, C.G. Christodoulou, M. Georgiopoulos, "Radial basis function neural network algorithm for adaptive beamforming in cellular communication systems" Conference on Antennas and Propagation for Wireless Communications, Waltham, MA USA, Nov. 1998, pp.53-56

[3] C.Q. Xu, C.L. Law, S. Yoshida, "On nonlinear beamforming for interference cancellation" Vehicular Technology Conference, Vol.1, Rhodes, Greece, May 2001, pp.23-27

[4] A.M.G. Guerreiro, A.D.D. Neto, F.A. Lisboa, "A joint operation of a neural network and a GSC structure applied to linear and planar arrays" Microwave and Optoelectronics Conference, Vol.2, Rio de Janeiro, Brazil, Aug. 1999, pp.493-498

[5] L. Hanzo, C. H. Wong, M. S. Yee: Adaptive Wireless Transceivers: Turbo-Coded, Turbo-Equalized and Space-Time Coded TDMA, CDMA, and OFDM Systems, John Wiley and IEEE Press, Feb. 2002

[6] S. Chen and B. Mulgrew, "Overcoming Co-Channel Interference Using an Adaptive Radial Basis Function Equalizer." Signal Processing (EURASIP), Vol.28, No.1, July 1992, pp:91-107

[7] A. Yener, R.D. Yates, S. Ulukus, "Combined multiuser detection and beamforming for CDMA systems: filter structures." IEEE Transactions on Vehicular Technology, Vol.51, Sept. 2002, pp.1087-1095

[8] C. Tidestav, M. Sternad, A. Ahlen, "Reuse within a cell-interference rejection or multiuser detection?" IEEE Transactions on Communications, Vol.47, No.10, Oct. 1999, pp.1511-1522 